\title{
Closure of the Equine Nephrosplenic Space Using a Single LED Powered Trocar via Standing Mini-Laparotomy
}

\author{
Christian Bussy ${ }^{1 *}$, Kossay Benredouane ${ }^{1}$, Juan Munoz ${ }^{2}$, Diane Petat ${ }^{1}$ \\ ${ }^{1}$ Clinique Vétérinaire du Grand Renaud, Saint Saturnin, France \\ ${ }^{2}$ The Department of Surgery, Universidad Alfonso X el Sabio, Madrid, Spain \\ Email: *christian.bussy@vetoequin.fr
}

How to cite this paper: Bussy, C., Benredouane, K., Munoz, J. and Petat, D. (2019) Closure of the Equine Nephrosplenic Space Using a Single LED Powered Trocar via Standing Mini-Laparotomy. Open Journal of Veterinary Medicine, 9, 11-20. https://doi.org/10.4236/ojvm.2019.92002

Received: January 21, 2019

Accepted: February 22, 2019

Published: February 25, 2019

Copyright ( 2019 by author(s) and Scientific Research Publishing Inc. This work is licensed under the Creative Commons Attribution International License (CC BY 4.0).

http://creativecommons.org/licenses/by/4.0/ (c) (i) Open Access

\begin{abstract}
Objective: To evaluate a technique using a custom-developed LED powered Single Port Trocar for closure of the nephrosplenic space via a standing mini-laparotomy. Design: Prospective case series. Animals: 60 client-owned horses with a history of nephrosplenic entrapment. Procedures: The nephrosplenic space was closed under direct visualization through a specifically designed LED powered Single Port Trocar in standing sedated horses having experienced recurrence or previous surgical correction of a nephrosplenic entrapment. Obliteration of the space was performed in a cranial to caudal direction in a simple continuous fashion. Time required for suturing the nephrosplenic space, as well as per and post-operative complications were recorded. Clients and referring veterinarians were asked to communicate any complication that occurred after discharge. Results: Surgical time ranged from 23 to $45 \mathrm{~min}$. No complication except for self-limited spleen capsule bleeding occurred during the procedure. Minor wound discharge was noted in 2 cases, and another 2 horses developed post-surgical sweating patches close to the incision. All horses were allowed to progressively resume exercise, starting 1 month post discharge from the hospital. Cosmetic results were considered satisfactory and no recurrence of left dorsal displacement of the colon was reported post-surgery. Conclusions and clinical relevance: A Single Port LED powered Trocar is a technique that allows effective, fast, and safe closure of the nephrosplenic space in the standing horse with minimal instrumentation.
\end{abstract}

\section{Keywords}

Colic, Colon, Nephrosplenic Entrapment, Laparotomy 


\section{Introduction}

Left dorsal displacement of the large colon (LDDLC), or nephrosplenic entrapment, occurs when the ascending (left) colon displaces dorsally in the nephrosplenic space, over its ligament [1] [2] [3], with a reported incidence ranging from $6 \%$ to $9 \%$ of colic cases [2] [3] [4]. A reported recurrence rate ranging from $3.2 \%$ to $23 \%$ [1] [3] [4] [5], with an individual incidence up to 5 times [4], advocates preventive closure of the nephrosplenic space, which has been shown to prevent LDDLC recurrence and significantly decrease colic scores in the long term [3] [6]. The procedure can be performed through under general anesthesia or standing through a flank incision with resection of the last rib [5] [7]. Another standing laparoscopic technique was described by Marien and others with improved visualization and post-operative cosmetic appearance, and less convalescence time and post-operative morbidity [6] [8]-[13]. This procedure, although now considered a standard, requires specialized equipment and training with a high learning curve for developing proficiency [14], mostly depending on developing specific laparoscopic experience [8]. Small diameter needles used for standard laparoscopic nephrosplenic space closure can cause tearing of the spleen during suturing [4] [9], and techniques using custom-made cannulas to accept needle holders and larger needles have been described [6] [8]. Hand-assisted procedures through a mini laparotomy may be more straightforward for a surgeon less versed in performing laparoscopy, but they are more traumatic and less tolerated by the standing sedated horse [13]. We thought that it was important to develop a technique that retained the advantage of being less-invasive, but that could be performed without specialized training. The purpose of this manuscript is to describe the technique and the equipment developed and used for that technique, and to report on the results of a large number of horses treated with this technique.

\section{Materials and Methods}

\subsection{Animals}

Horses and ponies with a history of two or more occurrences of medically treated or one surgically resolved nephrosplenic entrapment were enrolled in the study, following the current recommendations [2] [3] [6]. There were 24 geldings, 24 mares and 12 stallions, between 3 and 20 years old (mean age 8 years old), mostly warmbloods (70\%), weighting approximatively between 320 and $580 \mathrm{~kg}$.

After explanation of the different procedures available, informed owner consent was obtained prior to surgery. The procedure was only a modification/combination of the currently used techniques, both the flank laparotomy, commonly used for uterine torsions, and the laparoscopic closure of the nephrosplenic space. Animal welfare was respected by minimizing surgery time and potential discomfort by the use of adequate sedation, local anesthesia and pain killers prior to and after surgery, as well as close monitoring of horse behavior 
after the procedure.

\subsection{Preoperative Preparation}

Whenever possible, forage feeding was stopped for $48 \mathrm{~h}$ and replaced by a complete pelleted food prior to surgery to reduce the volume of the large intestine contents. When the procedure was performed immediately after medical resolution of an episode of nephrosplenic entrapment, food was withheld for a minimum of $12 \mathrm{~h}$ prior to surgery. A complete blood count and biochemistry profile were performed. All horses were administered penicillin $\mathrm{G}$ procaine $(22,000 \mathrm{IU} / \mathrm{kg}$, IM, BID 4 days), and flunixin meglumine $(1.1 \mathrm{mg} / \mathrm{kg}$, IV, BID for 2 days, then SID 2 days). Tetanus vaccination status was checked and a tetanus toxoid booster administered when needed. A rectal palpation was performed prior to surgery to assess the size of the large intestine and confirm that the nephrosplenic space was free.

\subsection{Surgical Procedure}

A pilot study performed on cadavers with owners written consent helped develop the instrumentation for a single port approach with a LED-illuminated Trocar $^{1}$, and investigate adequate anatomical landmarks, needle and suture types. The Trocar has an ovoid, tapered, cross-section with external dimensions of 4 by $6 \mathrm{~cm}$ at its intra-abdominal extremity and $5 \times 7.5 \mathrm{~cm}$ at its external end, equipped with a handle perpendicular to the its shaft. A row of LEDs for illumination of the abdominal cavity is incorporated at the intra-abdominal extremity, powered by 2 AA batteries lodged in the handle, making the Trocar wireless (Figure 1).

Clinically affected horses were restrained in standing stocks, tail tied up, and given head support. The left paralumbar fossa was clipped from the last intercostal space to $20 \mathrm{~cm}$ caudally to the tuber coxae, and from $20 \mathrm{~cm}$ dorsally to the

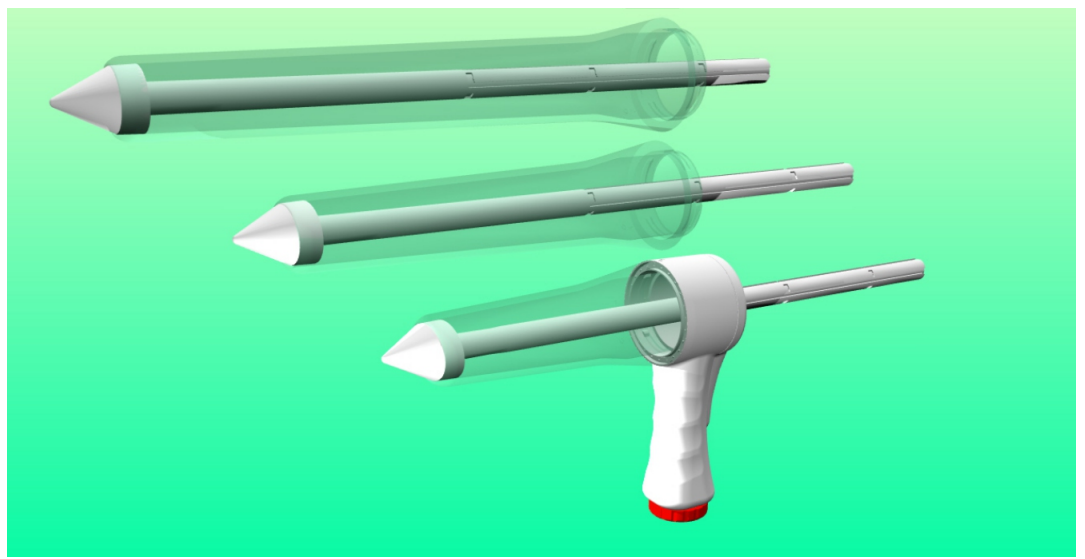

Figure 1. Drawing of the LED-powered trocar assembly with its translucent body of different lengths, the vertical handle through which the batteries are inserted, and the removable conical trocar for insertion.

${ }^{1}$ Trocar GR, GR VET Innovation, Saint-Saturnin, 72, France. 
lumbar vertebrae transverse process to the fold of flank level. The area was then aseptically prepared with a povidone iodine scrub followed by alcohol. The horse was sedated for the procedure using detomidine $(0.01 \mathrm{mg} / \mathrm{kg}, \mathrm{IV})$ and butorphanol $(0.01 \mathrm{mg} / \mathrm{kg}, \mathrm{IV})$. Another dose of detomidine and butorphanol was given during the surgery as needed based on the horse's level of awareness. Lidocaine was used to provide local anesthesia with $15 \mathrm{~mL}$ injected subcutaneously [SC] at the proposed incision site, and another $15 \mathrm{~mL}$ infiltrated into the muscles planes down to the peritoneum. A sterile table drape $(160 \mathrm{~cm} \times 200 \mathrm{~cm})$ with a $15 \mathrm{~cm} \times 7 \mathrm{~cm}$ surgical opening was centered over the surgical site and secured using cutaneous staples.

A left vertical paralumbar incision was performed, starting $5 \mathrm{~cm}$ ventral to the lumbar transverse processes, equidistant between the $18^{\text {th }}$ rib and the cranial edge of the tuber coxae, and extending ventrally 8 to $10 \mathrm{~cm}$. Following the incision of the skin and the subcutaneous tissues, a "Modified Grid Technique" was used to incise the abdominal musculature. The external abdominal oblique muscle was first incised along the line of the skin incision followed by the blunt separation of the internal abdominal oblique and transversus abdominis muscles along the direction of their fibers. Hemostasis was performed as necessary. Separation of the internal abdominal oblique muscle was performed with care in order not to severe the deep circumflex iliac artery. Once exposed, the peritoneum was punctured using blunt scissors and opened with either scissors or fingers.

The trocar was then inserted through the incision, perpendicular to the muscle wall, and its obturator removed. Once in the abdominal cavity, the trocar was directed cranially in order to visualize the spleen and the left kidney (Figure 2).

Upon entering the abdominal cavity, if a loop of small intestine or floating

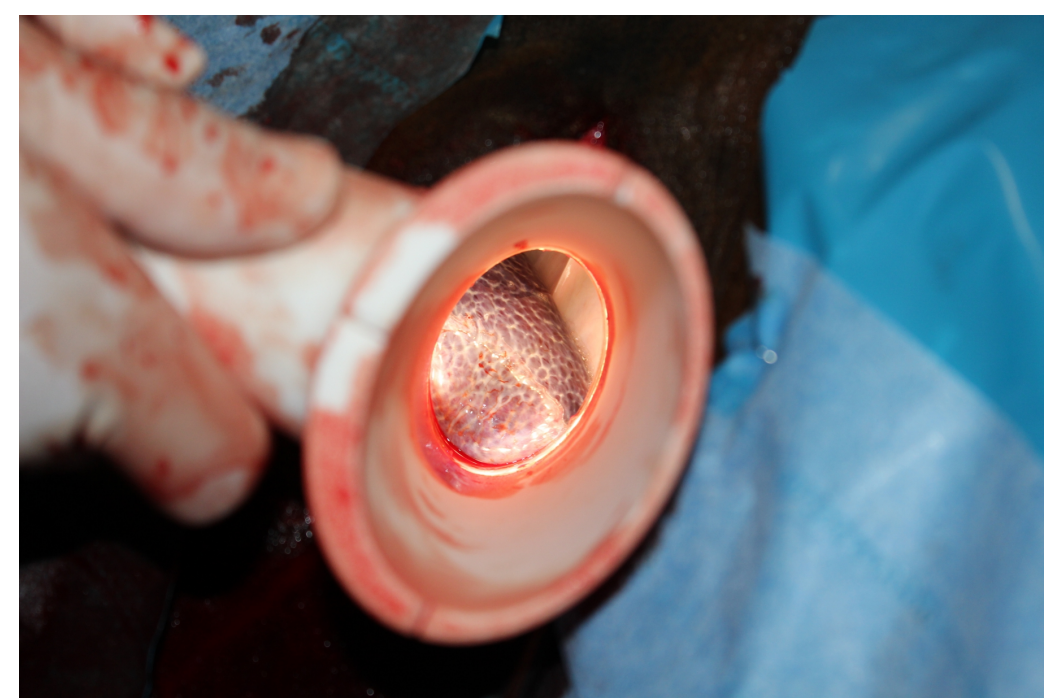

Figure 2. View of the nephrosplenic space through the trocar. The handpiece is held on the left of the picture; the spleen is seen on the left and lower aspect of the trocar window, while the renal capsule appears as the light pink smooth vertical surface on the upper right corner. 
colon was found within the nephrosplenic space, it was pushed using a long instrument such as a needle holder placed through the Trocar. If the large colon was entrapped in the nephrosplenic space, the Trocar was removed, the surgical incision slightly lengthened, and the nephrosplenic entrapment reduced manually [13] [15].

A $240 \mathrm{~cm}$ long USP 2 Lactomer 9-1 loop suture mounted on a $65 \mathrm{~mm} \mathrm{1/2}$ curve, taper point needle ${ }^{2}$ was used for suturing. Using a specially designed 50 $\mathrm{cm}$ working length needle holder, the needle was introduced into the abdominal cavity and positioned at the craniolateral aspect of the left kidney capsule, approximately $10 \mathrm{~cm}$ proximal to the cranial edge of the nephrosplenic ligament. The needle was directed dorsoventrally in an almost vertical direction, ensuring that only the fibrous capsule of the kidney was included in the suture, with minimal involvement of the renal cortex. Once the needle was passed through the capsule of the kidney, it was inserted from the medial aspect of the spleen, $4 \mathrm{~cm}$ distal to its dorsal limit. The $1 / 2$ curve needle was advanced latero-dorsally such as to exit the spleen at a higher level than its entry point. The needle was grasped using the needle-holder, pulled out of the abdomen, passed inside the loop and tension was applied by sliding the loop toward the suture's starting point, avoiding the necessity to apply a first knot. The nephrosplenic space was then closed with a simple continuous pattern, progressing until approximately $20 \mathrm{~cm}$ caudally from its point of entry depending on the size of the spleen (Figure 3).

The continuous suture pattern was then stopped as follows: one of the threads from the loop was cut at the level of the needle and kept manually under tension

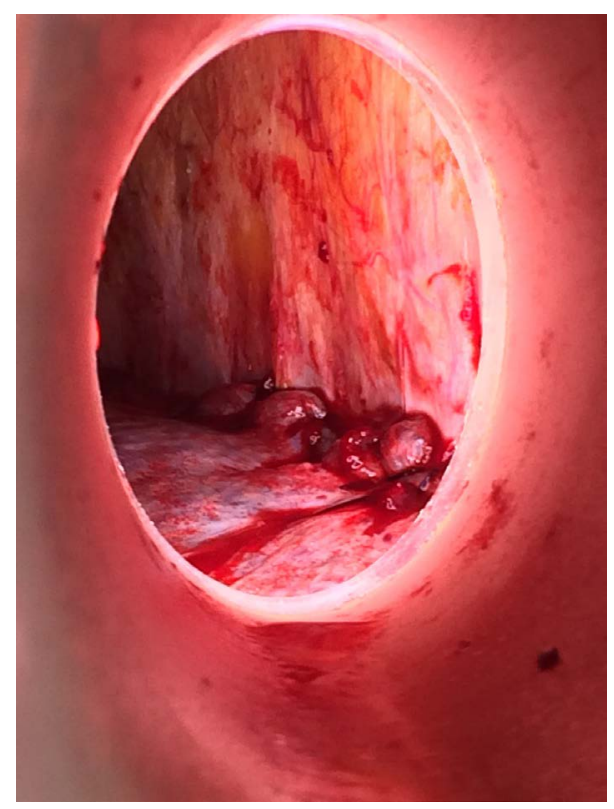

Figure 3. View of the sutured nephrosplenic space at the end of the procedure, through the trocar: the renal capsule is seen as a vertical surface on the upper aspect of the picture, and the spleen capsule as a vertical surface at its lower aspect. The sutures closely appose one capsule to the other without visible tearing.

${ }^{2}$ Polysorb, Covidien France SAS, Les Ulis, 91, France. 
in an extra-abdominal position while another bite was taken with the needle and its single thread between the kidney and the spleen. Outside the Trocar, the needle was removed and a simple knot (half key, extracorporeal) was placed between the two threads using the knot-pusher, which enabled the sliding of the knot up to the spleen. This technique, simple knot pushed to the spleen with the $50 \mathrm{~cm}$ knot-pusher each time, was repeated 4 times. Close attention was paid to the change of thread direction each time to make sure the knots crossed each other and to make sure they did not get loose. The remaining thread was cut under the needle, inserted into a hole within the head of the $50 \mathrm{~cm}$ knot pusher, with sharp edges designed to cut the thread when put under tension, and cut approximately $1 \mathrm{~cm}$ to the last knot made; the same was done with the second thread.

Once all the knots were completed and checked, the Trocar was removed. A $\mathrm{N}^{\circ} 2$ polyglactin $910^{3}$ suture on a $48 \mathrm{~mm} 1 / 2$ curve taper point needle, was used to close the surgical incision in two layers. The first layer consisted of a simple continuous pattern that included the peritoneum, transverse and internal abdominal oblique muscles. The second layer consisted of a simple continuous pattern including the external abdominal oblique muscle and the subcutaneous tissue. Staples were used to close the skin.

Two different surgeons performed the procedures but the same technician was present for all surgeries.

\subsection{Post-Operative Care and Outcome}

Horses were monitored twice daily for any sign of pain, increase of heart or respiratory rate, incision swelling or pain, and appetite until discharge, which occurred between 3 and 5 days post-surgery. Antibiotics and NSAIDs were continued for 3 days post-operatively (penicillin G procaine 22,000 IU/kg, IM, BID and flunixin meglumine $1.1 \mathrm{mg} / \mathrm{kg}, \mathrm{IV}, \mathrm{BID}$ for 1 day, then SID 2 days) and the cutaneous staples were removed around 2 weeks after surgery.

During the first 8 days, horses were stall rested and hand-walked 10 minutes twice daily, and then kept in a small paddock for an additional 20 days. In most cases, exercise was reintroduced one month following the surgical procedure. Three horses underwent second-look laparoscopy a month after the procedure.

\section{Results}

Sixty horses underwent the procedure within a 4 years' period, some owners elected to have the surgery performed after one episode of nephrosplenic entrapment to avoid recurrence and potential disruption of their competitive season. Sedation proved to be adequate and horses were stable did not show obvious signs of pain during the procedure, except for slight discomfort at peritoneal puncture. No complication other than minor and self-limited bleeding from the spleen capsule occurred during surgery. Visualization of the area of interest was never compromised by hemorrhage and proved excellent in all cases despite ${ }^{3}$ Vicryl, Ethicon, Issy-les-Moulineaux, 92, France. 
wide variations in nephrosplenic space anatomy. In horses operated on within days of an actual entrapment, the spleen subjectively appeared engorged and in most cases, if owners agreed, the procedure was postponed to a later date to avoid this complication, which made the suturing more delicate due to the thickness of the upper edge of the spleen. Two horses presented to the clinic for an elective closure of the nephrosplenic space had an undiagnosed nephrosplenic entrapment of the large colon [13]. In those cases, the incision was slightly enlarged to allow for manual correction of the entrapment. The Trocar was then placed into the surgical incision and suturing of the nephrosplenic space was performed. A third case had a previously failed laparoscopic nephrosplenic space closure. The horse was showing signs compatible with recurrent obstruction of the small intestine, possibly adhesions. The horse underwent exploratory laparotomy through the linea alba to break down the adhesions from the nephrosplenic space. A few weeks following the laparotomy, closure of the nephrosplenic space through the flank with the help of the single port Trocar was easily performed.

The average time needed to complete each surgical step and the total surgical time from incision to closure ranged from 23 to $45 \mathrm{~min}$. A mild post-surgical edema at the incision site that resolves by itself within 3 to 4 days was generally observed. Two horses produced a seroma that drained rapidly after staples from the distal part of the incision were removed, and another 2 showed a patch of sudation close to the incision area within weeks after the procedure.

In all 3 cases where second laparoscopy was performed (based on owner's concerns about efficiency of the procedure before it became accepted), the nephrosplenic space was found to be properly closed and healed with no signs of spleen or kidney tearing.

\section{Discussion}

Classic laparoscopic nephrosplenic closure offers the advantages of a minimally invasive surgery performed on a standing horse, and a good 2-dimensional view of the surgical site. However, available laparoscopic instrumentation is often under-sized for large individuals, the needles often too small, which can lead to capsule tearing and potentially subsequent intestinal adhesions as illustrated in one of our cases, and the classic instruments length insufficient when used in larger horses.

Distension of the abdomen with $\mathrm{CO}_{2}$, commonly used during closure of the nephrosplenic space, and which can in some cases cause discomfort during and for several days after surgery [16], is not required for a single port trocar procedure, eliminating issues arising from loss of distension, and potential irritation.

Authors reported complications such as visceral punctures of the large intestine or pneumothorax formation when introducing the laparoscope between the 2 last floating ribs [16] [17], and the procedure remains challenging even to experimented surgeons. Complications such as visceral punctures or pneumothorax formation were never observed during the single port trocar procedure. The peritoneum puncture, performed under direct visualization with scissors, en- 
larged before inserting the trocar, prevented pushing the peritoneum away from the muscular wall, which could obstruct the view for the remaining of the surgery and is regularly encountered during laparoscopic placement.

The most significant advantage of the single port technique lies in its very short learning curve. The ovoid LED powered Trocar provides a 3-dimensional visualization of the nephrosplenic space, unaltered by the horse weight shifting, while allowing the surgeon to comfortably operate the needle holder and knot-pusher through the single port, which offers a significant advantage, especially for a laparoscopically untrained surgeon. This explains why surgical time (23 to $45 \mathrm{~min}$ in our study) compares favorably with published laparoscopic nephrosplenic space closure time of $30 \mathrm{~min}$ [4] [6], 30 - $65 \mathrm{~min}$ [8].

Finally, resolution of the entrapment and closure of the nephrosplenic space were both performed during the same procedure in 2 clinical cases, which would not have been possible via laparoscopy.

Disadvantages of the above described technique includes the necessity for a new piece of equipment (LED powered Trocar), but at least one less person is required when performing the surgery with the single port trocar, versus a classic laparoscopy, and the trocar and its instruments cost less than one tenth of the price of the complete equipment for a classic laparoscopy.

The longest incision compared to the laparoscopic technique could also be seen as a drawback, especially when aesthetics is of utmost concern, even though the scar would be mostly visible in horses with very thin coat or clipped, and the minimal tissue handling seems to favor first intention healing ${ }^{4}$. The slightly longer recovery time is probably not relevant in a clinical setting.

The present study is limited by the loss to follow-up of some horses, which are generally referred and therefore whose owners are more difficult to contact. Some individuals having recurrent colic medically treated might have suffered from recurrent displacement, without being precisely diagnosed, and some might have been euthanized without a definite diagnosis nor autopsy, limiting the long-term evaluation of the technique. No foals nor miniature horses were included in the study and therefore the feasibility of the above described technique still needs to be evaluated on those, especially in foals who might be more fractious to standing surgery. Those limitations are however comparable that found with the classic laparoscopic technique.

The single Trocar technique therefore appears a safe alternative technique to the classic laparoscopy technique to close the nephrosplenic space.

\section{Acknowledgements}

No external funding was used in this study. The first author developed the Trocar GR and is a partner in Gr Vet Innovation, the commercializing company.

\section{Conflicts of Interest}

The authors declare no conflicts of interest regarding the publication of this paper. ${ }^{4}$ Dr David E. Freeman, personal communication 2015. 


\section{References}

[1] Hardy, J., Minton, M., Robertson, J.T., et al. (2000) Nephrosplenic Entrapement in the Horse: A Retrospective Study of 174 Cases. Equine Veterinary Journal, 32, 95-97. https://doi.org/10.1111/j.2042-3306.2000.tb05342.x

[2] Lindegaard, C., Ekstrom, C.T., Wulf, S.B., et al. (2011) Nephrosplenic Entrapment of the Large Colon in 142 Horses (2000-2009): Analysis of Factors Associated with Decision of Treatment and Short Term Survival. Equine Veterinary Journal, 43, 63-68. https://doi.org/10.1111/j.2042-3306.2011.00376.x

[3] Nelson, B.B., Ruple-Czerniak, A.A., Hendrickson, D.A., et al. (2016) Laparoscopic Closure of the Nephrosplenic Space in Horses with Nephrosplenic Colonic Entrapment: Factors Associated with Survival and Colic Recurrence. Veterinary Surgery, 45, 60-69. https://doi.org/10.1111/vsu.12549

[4] Rocken, M., Schubert, C., Mosel, G., et al. (2005) Indications, Surgical Technique, and Long Term Experience with Laparoscopic Closure of the Nephrosplenic Spec in Standing Horses. Veterinary Surgery, 34, 637-641. https://doi.org/10.1111/j.1532-950X.2005.00098.x

[5] Huskamp, B. and Kopf, N. (1980) Die Verlagerung des Colon ascendens in den Milz-Nieren-Raum beim Pferd (2). Tierarztl Prax, 8, 495-506.

[6] Farstvedt, E. and Hendrickson, D. (2005) Laparoscopic Closure of the Nephrosplenic Space for Prevention of Recurrent Nephrosplenic Entrapment of the Ascending Colon. Veterinary Surgery, 34, 642-645. https://doi.org/10.1111/j.1532-950X.2005.00099.x

[7] Zekas, L.J., Ramirez, S. and Brown, M.P. (1999) Ablation of the Nephrosplenic Space for Treatment of Recurring Left Dorsal Displacement of the Large Colon in a Racehorse. Journal of the American Veterinary Medical Association, 214, 1361-1363.

[8] Albanese, V., Hanson, R.R., McMaster, M.A., et al. (2016) Use of a Barbed Knotless Suture for Laparoscopic Ablation of the Nephrosplenic Space in 8 Horses. Veterinary Surgery, 45, 824-830. https://doi.org/10.1111/vsu.12520

[9] Bracamonte, J.L. and Duke-Novakovski, T. (2016) A Pilot Study Evaluating Laparoscopic Closure of the Nephrosplenic Space Using an Endoscopic Suturing Device in Standing Horses. Canadian Veterinary Journal, 57, 651-54.

[10] Burke, M.J. and Parente, E.J. (2016) Prosthetic Mesh for Obliteration of the Nephrosplenic Space in Horses: 26 Clinical Cases. Veterinary Surgery, 45, 201-207. https://doi.org/10.1111/vsu.12434

[11] Epstein, K.L. and Parente, E.J. (2006) Laparoscopic Obliteration of the Nephrosplenic Space Using Polypropylene Mesh in Five Horses. Veterinary Surgery, 35, 431-437. https://doi.org/10.1111/j.1532-950X.2006.00171.x

[12] Marien, T., Adriaenssen, A., Hoeck, F.V., et al. (2001) Laparoscopic Closure of the Nephrosplenic Space in Standing Horses. Veterinary Surgery, 30, 559-563. https://doi.org/10.1053/jvet.2001.28436

[13] Munoz, J. and Bussy, C. (2013) Standing Hand Assisted Laparoscopic Treatment of the Left Dorsal Displacement of the Large Colon and Closure of the Nephrosplenic Space. Veterinary Surgery, 42, 595-599. https://doi.org/10.1111/j.1532-950X.2012.01050.x

[14] Pope, J.F. and Knowles, T.G. (2014) Retrospective Analysis of the Learning Curve Associated with Laparoscopic Ovariectomy in Dogs and Associated Perioperative Complication Rates. Veterinary Surgery, 43, 668-677.

https://doi.org/10.1111/j.1532-950X.2014.12216.x 
[15] Krueger, C.R. and Klohnen, A. (2015) Surgical Correction of Nephrosplenic Entrapment of the Large Colon in 3 Horses via Left Flank Laparotomy. Veterinary Surgery, 44, 392-397. https://doi.org/10.1111/j.1532-950X.2014.12293.x

[16] Hendrickson, D.A. (2008) Complications of Laparoscopic Surgery. Veterinary Clinics of North America, 24, 557-571.

[17] Shettko, D.L. (2000) Complications in Laparoscopic Surgery. Veterinary Clinics of North America, 16, 377-383. 